WUE-ITP-98-043

SPhT-T98/019

\title{
Perturbative QCD Correction to the Light-Cone Sum Rule for the $B^{*} B \pi$ and $D^{*} D \pi$ Couplings
}

\author{
A. Khodjamirian ${ }^{1, a}$, R. Rückl ${ }^{1}$, S. Weinzierl ${ }^{2, b}$, O. Yakovlev $^{1}$ \\ ${ }^{1}$ Institut für Theoretische Physik, Universität Würzburg, D-97074 Würzburg, Germany \\ 2 Service de Physique Théorique, Centre d'Etudes de Saclay, \\ F-91191 Gif-sur-Yvette Cedex, France
}

\begin{abstract}
The $B^{*} B \pi$ and $D^{*} D \pi$ couplings have previously been derived from a QCD lightcone sum rule in leading order. Here, we describe the calculation of the $O\left(\alpha_{s}\right)$ correction to the twist 2 term of this sum rule. The result is used for a first nextto-leading order analysis. We obtain $g_{B^{*} B \pi}=22 \pm 7$ and $g_{D^{*} D \pi}=10.5 \pm 3$, where the error indicates the remaining theoretical uncertainty.
\end{abstract}

${ }^{a}$ On leave from Yerevan Physics Institute, 375036 Yerevan, Armenia

${ }^{b}$ now at NIKHEF, P.O. Box 41882, 1009-DB Amsterdam, The Netherlands 
1. The hadronic $B^{*} B \pi$ coupling is defined by the on-shell matrix element

$$
\left\langle\bar{B}^{* 0}(p) \pi^{-}(q) \mid B^{-}(p+q)\right\rangle=-g_{B^{*} B \pi}(q \cdot \epsilon),
$$

where the meson four-momenta are given in brackets and $\epsilon_{\mu}$ is the polarization vector of the $B^{*}$. An analogous definition holds for the $D^{*} D \pi$ coupling. These couplings play an important role in $B$ and $D$ physics. For example, they determine the magnitude of the weak $B \rightarrow \pi$ and $D \rightarrow \pi$ form factors at zero pion recoil, i.e., at momentum transfer squared close to $\left(m_{B}-m_{\pi}\right)^{2}$ and $\left(m_{D}-m_{\pi}\right)^{2}$, respectively. Moreover, the coupling constant $g_{D^{*} D \pi}$ is directly related to the decay width of $D^{*} \rightarrow D \pi$. The decay $B^{*} \rightarrow B \pi$ is kinematically forbidden.

Theoretically, the $B^{*} B \pi$ and $D^{*} D \pi$ couplings have been studied using a variety of methods]. Among these, QCD light-cone sum rules (LCSR) have proved particularly powerful. The first LCSR calculation of $g_{B^{*} B \pi}$ and $g_{D^{*} D \pi}$ including perturbative QCD effects in leading order (LO) was reported in [1]. It is well known, however, that in heavylight systems next-to-leading order (NLO) effects can be essential. An important example is provided by the heavy-to-light form factors [2, 3, , 4, 5]. This calls for a NLO analysis of $g_{B^{*} B \pi}$ and $g_{D^{*} D \pi}$.

In this letter, we describe the calculation of the $O\left(\alpha_{s}\right)$ correction to the leading twist 2 term of the relevant LCSR. The result is then used for a first NLO evaluation. We present numerical predictions for $g_{B^{*} B \pi}$ and $g_{D^{*} D \pi}$ together with an estimate of the remaining theoretical uncertainties.

2. For definiteness, we focus on the derivation of the $B^{*} B \pi$ coupling. For the $D^{*} D \pi$ coupling the procedure is completely analogous. The LCSR for $g_{B^{*} B \pi}$ is derived from the vacuum-to-pion correlation function of the vector and pseudoscalar currents involving the $b$-quark and light quark fields:

$$
\begin{gathered}
F_{\mu}(p, q)=i \int d^{4} x e^{i p x}\left\langle\pi^{-}(q)\left|T\left\{\bar{d}(x) \gamma_{\mu} b(x), m_{b} \bar{b}(0) i \gamma_{5} u(0)\right\}\right| 0\right\rangle \\
=F\left(p^{2},(p+q)^{2}\right) q_{\mu}+\tilde{F}\left(p^{2},(p+q)^{2}\right) p_{\mu} .
\end{gathered}
$$

Inserting in the above matrix element complete sets of intermediate hadronic states car-

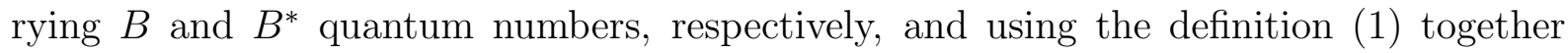
with

$$
m_{b}\left\langle B^{-}\left|\bar{b} i \gamma_{5} u\right| 0\right\rangle=m_{B}^{2} f_{B}
$$

and

$$
\left\langle 0\left|\bar{d} \gamma_{\mu} b\right| \bar{B}^{* 0}\right\rangle=m_{B^{*}} f_{B^{*}} \epsilon_{\mu},
$$

one obtains double dispersion relations for the invariant functions $F$ and $\tilde{F}$. In the following, we only need

$$
F\left(p^{2},(p+q)^{2}\right)=\frac{m_{B}^{2} m_{B^{*}} f_{B} f_{B^{*}} g_{B^{*} B \pi}}{\left(p^{2}-m_{B^{*}}^{2}\right)\left((p+q)^{2}-m_{B}^{2}\right)}+\iint_{\Sigma} \frac{\rho^{h}\left(s_{1}, s_{2}\right) d s_{1} d s_{2}}{\left(s_{1}-p^{2}\right)\left(s_{2}-(p+q)^{2}\right)} .
$$

\footnotetext{
${ }^{1}$ An overview is given, e.g., in Tab. 1 of ref. [1].
} 
The first term on the r.h.s. is the ground-state contribution involving the $B$ and $B^{*}$ masses, decay constants, and the desired $B^{*} B \pi$ coupling. The second term results from the higher resonances and continuum states in the $B^{*}$ and $B$ channels described by the hadronic spectral density $\rho^{h}\left(s_{1}, s_{2}\right)$. The integration boundary in the $\left(s_{1}, s_{2}\right)$-plane is denoted by $\Sigma$. In general, the dispersion relation (5) requires subtractions in order to render it finite. However, these subtraction terms being polynomials in $p^{2}$ and/or $(p+q)^{2}$ will be removed by Borel transformation $[$, and are therefore not made explicit here.

In QCD, the invariant amplitude $F\left(p^{2},(p+q)^{2}\right)$ can be calculated at $p^{2},(p+q)^{2} \ll m_{b}^{2}$ by expanding the time-ordered product of currents in the correlation function (2) near the light-cone, that is around $x^{2}=0$ (for reviews, see e.g. [6, 7]). The result is given by convolutions of hard scattering amplitudes and pion distribution amplitudes. The latter parameterize the long-distance effects and are classified by twist. This paper mainly deals with the leading twist 2 term

$$
F^{(2)}\left(p^{2},(p+q)^{2}\right)=-f_{\pi} \int_{0}^{1} d u \varphi_{\pi}(u, \mu) T\left(p^{2},(p+q)^{2}, u, \mu\right),
$$

$T$ being the hard amplitude, $\varphi_{\pi}$ the twist 2 distribution amplitude, and $\mu$ the factorization scale. In analogy to (5), we write (6) in the form of a double dispersion relation:

$$
F^{(2)}\left(p^{2},(p+q)^{2}\right)=\iint \frac{\rho\left(s_{1}, s_{2}\right) d s_{1} d s_{2}}{\left(s_{1}-p^{2}\right)\left(s_{2}-(p+q)^{2}\right)},
$$

where the corresponding spectral density $\rho$ is given by

$$
\rho\left(s_{1}, s_{2}\right)=\frac{1}{\pi^{2}} \operatorname{Im}_{s_{1}} \operatorname{Im}_{s_{2}} F^{(2)}\left(s_{1}, s_{2}\right) .
$$

After equating (5) and (7) and applying Borel transformation one obtains the basic sum rule

$$
\begin{gathered}
m_{B}^{2} m_{B^{*}} f_{B} f_{B^{*}} g_{B^{*} B \pi} \\
\exp \left(-\frac{m_{B^{*}}^{2}}{M_{1}^{2}}-\frac{m_{B}^{2}}{M_{2}^{2}}\right)+\iint_{\Sigma} \rho^{h}\left(s_{1}, s_{2}\right) \exp \left(-\frac{s_{1}}{M_{1}^{2}}-\frac{s_{2}}{M_{2}^{2}}\right) d s_{1} d s_{2} \\
=\iint \rho\left(s_{1}, s_{2}\right) \exp \left(-\frac{s_{1}}{M_{1}^{2}}-\frac{s_{2}}{M_{2}^{2}}\right) d s_{1} d s_{2}
\end{gathered}
$$

with $M_{1}^{2}$ and $M_{2}^{2}$ being the Borel parameters associated with $p^{2}$ and $(p+q)^{2}$, respectively.

To proceed, one has to subtract from (9) the unknown contribution of the excited $B$ and $B^{*}$ states and of the continuum states. This can be achieved approximately by using quark-hadron duality. To this end, the integral over $\rho^{h}$ is replaced by a corresponding integral over $\rho$ with a suitable integration boundary $\widetilde{\Sigma}$. After subtraction, one has

$$
f_{B} f_{B^{*}} g_{B^{*} B \pi}=\frac{1}{m_{B}^{2} m_{B^{*}}} \int^{\widetilde{\Sigma}} \int\left(s_{1}, s_{2}\right) \exp \left(\frac{m_{B^{*}}^{2}-s_{1}}{M_{1}^{2}}+\frac{m_{B}^{2}-s_{2}}{M_{2}^{2}}\right) d s_{1} d s_{2} .
$$

\footnotetext{
${ }^{2}$ For the definition of the Borel transformation see, e.g., ref. [1].
} 
It should be noted that this LCSR actually determines the product of the $B$ and $B^{*}$ decay constants and the $B^{*} B \pi$ coupling. This will play an important role in the numerical analysis.

3. The perturbative hard scattering amplitude $T$ introduced in (6) can be expanded in a power series in $\alpha_{s}$ :

$$
T\left(p^{2},(p+q)^{2}, u, \mu\right)=T_{0}\left(p^{2},(p+q)^{2}, u\right)+\frac{\alpha_{s} C_{F}}{4 \pi} T_{1}\left(p^{2},(p+q)^{2}, u, \mu\right)+O\left(\alpha_{s}^{2}\right) .
$$

The lowest-order term is given by [1]

$$
T_{0}\left(p^{2},(p+q)^{2}, u\right)=-\frac{m_{b}^{2}}{m_{b}^{2}-p^{2}(1-u)-(p+q)^{2} u},
$$

where $m_{b}$ is defined to be pole mass of the $b$ quark. The $O\left(\alpha_{s}\right)$ term $T_{1}$ has recently been calculated [2, 3] in the context of the LCSR determination of the $B \rightarrow \pi$ form factor $f^{+}\left(p^{2}\right)$. For brevity, we do not repeat the explicit expressions here. They can be found in [2] together with a detailed discussion of the treatment of ultraviolet and collinear divergences.

The pion distribution amplitude $\varphi_{\pi}$ is associated [8, 9] with the leading twist 2 term of the matrix element p

$$
\left\langle\pi(q)\left|\bar{u}(x) \gamma_{\mu} \gamma_{5} d(0)\right| 0\right\rangle=-i q_{\mu} f_{\pi} \int_{0}^{1} d u \varphi_{\pi}(u, \mu) e^{i u q \cdot x}+\ldots,
$$

where the ellipses stand for terms of higher twist. The scale dependence of $\varphi_{\pi}(u, \mu)$ follows from a Brodsky-Lepage evolution equation. In LO, the solution is given by

$$
\varphi_{\pi}(u, \mu)=\varphi_{\pi}^{a s}(u)\left(1+\sum_{n=1}^{\infty} a_{2 n}(\mu) C_{2 n}^{3 / 2}(2 u-1)\right),
$$

where $C_{2 n}^{3 / 2}$ are Gegenbauer polynomials and $a_{2 n}(\mu)$ are multiplicatively renormalizable expansion coefficients. While

$$
\varphi_{\pi}^{a s}(u)=6 u(1-u)
$$

is the asymptotic distribution amplitude, the sum in (14) represents non-asymptotic effects with $a_{2 n}(\mu)$ vanishing at $\mu \rightarrow \infty$. In NLO [10], the coefficients $a_{2 n}$ mix under renormalization because the Gegenbauer polynomials are no longer eigenfunctions of the evolution kernel. A complete NLO expression for $\varphi_{\pi}(u, \mu)$ can be found in [11].

The spectral density $\rho\left(s_{1}, s_{2}\right)$ needed in (10) is obtained in LO by substituting the hard amplitude (12) and the distribution amplitude (14) in (6) and taking the imaginary parts according to (8). In NLO, one has to add the $O\left(\alpha_{s}\right)$ hard scattering amplitude $T_{1}$ as specified in (11), and use the solution of the NLO evolution equation for $\varphi_{\pi}$. This yields

$$
\rho\left(s_{1}, s_{2}\right)=\rho_{0}\left(s_{1}, s_{2}\right)+\frac{\alpha_{s} C_{F}}{4 \pi} \rho_{1}\left(s_{1}, s_{2}\right)
$$

\footnotetext{
${ }^{3}$ Here, the light-cone gauge, $x^{\mu} A_{\mu}^{a}=0$, is adopted for the gluon field $A_{\mu}^{a}$.
} 
with

$$
\rho_{0,1}\left(s_{1}, s_{2}\right)=-f_{\pi} \frac{1}{\pi^{2}} \operatorname{Im}_{s_{1}} \operatorname{Im}_{s_{2}} \int_{0}^{1} d u \varphi_{\pi}(u, \mu) T_{0,1}\left(s_{1}, s_{2}, u, \mu\right) .
$$

As can be seen from (12), $T_{0}$ only has a single pole in $(p+q)^{2}$. Hence,

$$
\operatorname{Im}_{s_{2}} T_{0}\left(s_{1}, s_{2}, u\right)=-\pi \delta\left(1-\frac{s_{1}}{m_{b}^{2}}(1-u)-\frac{s_{2}}{m_{b}^{2}} u\right)
$$

making the convolution with $\varphi_{\pi}(u, \mu)$ very simple. After taking the imaginary part in $s_{1}$, one finds the formal expression for $\rho_{0}$ given in [1]. In contrast, $T_{1}$ also has cuts in $(p+q)^{2}$, in addition to the pole. Therefore, $\operatorname{Im}_{s_{2}} T_{1}$ contains both $\delta$ - and $\theta$-functions in $s_{2}$. In the following, we use the explicit form given in [2]. To obtain the $O\left(\alpha_{s}\right)$ correction $\rho_{1}\left(s_{1}, s_{2}\right)$ one has to convolute $\operatorname{Im}_{s_{2}} T_{1}$ with the products $\varphi_{\pi}^{a s} C_{2 n}^{3 / 2}$. This integration should be carried out analytically in order to be able to take the imaginary part in $s_{1}$. In principle, this is possible, by considering the integrals of $u^{k} \operatorname{Im}_{s_{2}} T_{1}$ for each power $k$ separately. The calculation is rather cumbersome, but fortunately not really necessary. It turns out that the non-asymptotic effects in $\rho_{1}$ are much smaller than the overall theoretical uncertainty in the LCSR (10). It is therefore sufficient to calculate $\rho_{1}$ using the asymptotic pion distribution amplitude, unless the theoretical accuracy is increased dramatically.

With the above justification, we single out the asymptotic part of $\varphi_{\pi}$ given in (15) and perform the integral

$$
\int_{0}^{1} d u \varphi_{\pi}^{a s}(u) \operatorname{Im}_{s_{2}} T_{1}\left(s_{1}, s_{2}, u, \mu\right)
$$

analytically. After taking the imaginary part in $s_{1}$ we find

$$
\begin{gathered}
\rho_{1}^{a s}\left(s_{1}, s_{2}\right)=-f_{\pi} \frac{1}{\pi^{2}} \operatorname{Im}_{s_{1}} \operatorname{Im}_{s_{2}} \int_{0}^{1} d u \varphi_{\pi}^{a s}(u) T_{1}\left(s_{1}, s_{2}, u, \mu\right) \\
=f_{\pi} \frac{(r+1)^{2}}{\sigma}\left[\left(2 \pi^{2}+3 \ln \left(\frac{\sigma}{2}\right) \ln \left(1+\frac{\sigma}{2}\right)\right.\right. \\
\left.-\frac{3\left(3 \sigma^{3}+22 \sigma^{2}+40 \sigma+24\right)}{2(2+\sigma)^{3}} \ln \left(\frac{\sigma}{2}\right)+6 \operatorname{Li}_{2}\left(-\frac{\sigma}{2}\right)+\frac{3\left(\sigma^{2}+12 \sigma+12\right)}{4(2+\sigma)^{2}}\right) \delta(1-r) \\
\left.-6 \frac{r}{1+r}\left(\frac{\sigma}{1+r+\sigma}-2 \ln r+\ln \left(\frac{1+r(1+\sigma)}{1+r+\sigma}\right)\right) \frac{d^{3}}{d r^{3}} \ln |1-r|\right],
\end{gathered}
$$

where

$$
\sigma=\frac{s_{1}}{m_{b}^{2}}+\frac{s_{2}}{m_{b}^{2}}-2, \quad r=\frac{s_{1}-m_{b}^{2}}{s_{2}-m_{b}^{2}}
$$

and $\operatorname{Li}_{2}(x)=-\int_{0}^{x} \frac{d t}{t} \ln (1-t)$ is the Spence function. It is interesting to note that $\rho_{1}^{a s}$ contains no $\ln \mu$ terms. This is because in $T_{1}$ the coefficient of $\ln \mu$ is given by a convolution 
of $T_{0}$ with the LO kernel of the Brodsky-Lepage evolution equation [2]. It thus has to vanish when this kernel is folded with the asymptotic distribution amplitude $\varphi_{\pi}^{a s}$.

Finally, the resulting spectral density (16) is to be inserted in the LCSR (10). For $M_{1}^{2}=M_{2}^{2}=2 M^{2}$, the leading term coming from $\rho_{0}$ is simply proportional to $\varphi_{\pi}(1 / 2, \mu)$ as shown in [国]. Moreover, the precise form of the duality boundary $\widetilde{\Sigma}$ is irrelevant in this case since only the integration limits at $s_{1}=s_{2}$ enter. The NLO correction arising from $\rho_{1}$, on the other hand, depends on the shape of the integration boundary $\widetilde{\Sigma}$. We have chosen the triangle $s_{1}+s_{2}=2 s_{0}^{B}, s_{0}^{B}$ denoting the threshold of excited and continuum states in the $B$-channel. This choice allows to carry out the integration over the variable $r$ defined in (21) analytically. The final LCSR reads

$$
\begin{array}{r}
f_{B} f_{B^{*}} g_{B^{*} B \pi}=\frac{m_{b}^{2} f_{\pi}}{m_{B}^{2} m_{B^{*}}} e^{\frac{m_{B}^{2}+m_{B^{*}}^{2}}{2 M^{2}}}\left[M^{2}\left(e^{-\frac{m_{b}^{2}}{M^{2}}}-e^{-\frac{s_{0}^{B}}{M^{2}}}\right) \varphi_{\pi}(1 / 2, \mu)\right. \\
\left.+\frac{\alpha_{s} C_{F}}{4 \pi} \int_{2 m_{b}^{2}}^{2 s_{0}^{B}} f\left(\frac{s}{m_{b}^{2}}-2\right) e^{-\frac{s}{2 M^{2}}} d s+F^{(3,4)}\left(M^{2}, m_{b}^{2}, s_{0}^{B}, \mu\right)\right]
\end{array}
$$

with

$$
\begin{aligned}
f(x)= & \frac{\pi^{2}}{4}+3 \ln \left(\frac{x}{2}\right) \ln \left(1+\frac{x}{2}\right)-\frac{3\left(3 x^{3}+22 x^{2}+40 x+24\right)}{2(2+x)^{3}} \ln \left(\frac{x}{2}\right) \\
& +6 \operatorname{Li}_{2}\left(-\frac{x}{2}\right)-3 \operatorname{Li}_{2}(-x)-3 \operatorname{Li}_{2}(-x-1)-3 \ln (1+x) \ln (2+x) \\
& -\frac{3\left(3 x^{2}+20 x+20\right)}{4(2+x)^{2}}+\frac{6 x(1+x) \ln (1+x)}{(2+x)^{3}} .
\end{aligned}
$$

In (22), we have added the contributions $F^{(3,4)}$ from the pion distribution amplitudes of twist 3 and 4 . The twist 3 contribution is quantitatively as important as the twist 2 term, and has to be taken into account in phenomenological applications. Presently, $F^{(3,4)}$ is only known in LO [1]. The lack of knowledge of the NLO corrections hinders a complete NLO analysis.

The analogous LCSR for $f_{D} f_{D^{*}} g_{D^{*} D \pi}$ is obtained from (22) by formally replacing $b$ with $c, B$ with $D$, and $B^{*}$ with $D^{*}$.

4. The values of the parameters used in the numerical evaluation of the LCSR (22) and the two-point sum rules (2SR) for the corresponding decay constants are discussed in [1] and [2]. A brief recapitulation may therefore suffice here. For the $b$-quark mass and the corresponding continuum threshold we take

$$
m_{b}=4.7 \pm 0.1 \quad \mathrm{GeV}, \quad s_{0}^{B}=35 \mp 2 \quad \mathrm{GeV}^{2} .
$$

Variations of $m_{b}$ and $s_{0}^{B}$ within the allowed intervals are correlated by the stability requirement of $2 \mathrm{SR}$ as indicated by the opposite signs in (24). The renormalization scale of $\alpha_{s}$ is put equal to the factorization scale $\mu$ which will be fixed later. The running coupling constant is taken in two-loop approximation with $N_{f}=4$ and $\Lambda \frac{(4)}{M S}=315 \mathrm{MeV}$

\footnotetext{
${ }^{4}$ In NLO, they are given in [12].
} 
corresponding to $\alpha_{s}\left(m_{Z}\right)=0.118$ [13]. The Borel parameter in LCSR is constrained to the interval $6 \mathrm{GeV}^{2}<M^{2}<12 \mathrm{GeV}^{2}$, where the usual conditions of small $(<10 \%)$ twist 4 corrections and moderate $(<30 \%)$ contributions from heavier states are satisfied. In 2SR the allowed range is $2 \mathrm{GeV}^{2}<M^{2}<6 \mathrm{GeV}^{2}$. In the charm case, the corresponding parameters are given by

$$
m_{c}=1.3 \pm 0.1 \mathrm{GeV}, \quad s_{0}^{D}=6 \mp 1 \quad \mathrm{GeV}^{2},
$$

and $\Lambda \frac{(3)}{M S}=380 \mathrm{MeV}$. The ranges of the Borel parameter are $2 \mathrm{GeV}^{2}<M^{2}<4 \mathrm{GeV}^{2}$ in LCSR and $1 \mathrm{GeV}^{2}<M^{2}<2 \mathrm{GeV}^{2}$ in $2 \mathrm{SR}$. The meson masses are $m_{B}=5.279 \mathrm{GeV}$, $m_{B^{*}}=5.325 \mathrm{GeV}, m_{D}=1.87 \mathrm{GeV}, m_{D^{*}}=2.01 \mathrm{GeV}$, and $f_{\pi}=132 \mathrm{MeV}$. Finally, for the pion distribution amplitude at $u=0.5$ we adopt the value $\varphi_{\pi}(0.5, \mu)=1.2$ at $\mu=1$ $\mathrm{GeV}$ determined in 14 from the LCSR for the pion-nucleon coupling. This value is also consistent with the distribution amplitude $\varphi_{\pi}(u, \mu)$ used in [1, 2] to calculate the form factor $f^{+}$. The values of the vacuum condensates appearing in $2 \mathrm{SR}$ can be found in [6].
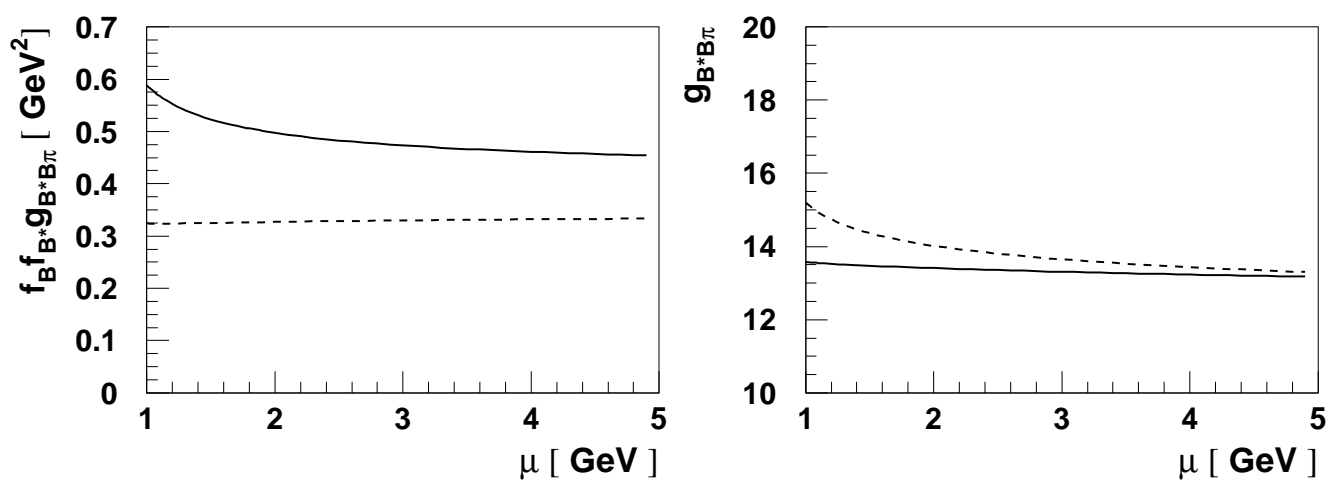

Figure 1: Scale dependence of the twist 2 term in the sum rule estimates of (a) $f_{B} f_{B^{*}} g_{B^{*} B \pi}$ and (b) $g_{B^{*} B \pi}$ : NLO (solid) in comparison to LO (dashed).

Let us first discuss the twist 2 term in the LCSR (22) being the main subject of this paper. This term is plotted in Fig. 1a as a function of $\mu$ for $M^{2}=8 \mathrm{GeV}^{2}$. As can be seen, the NLO correction is numerically large, increasing $f_{B} f_{B^{*}} g_{B^{*} B \pi}$ by about $50 \%$. It has the same sign as the NLO corrections to the $2 \mathrm{SR}$ for $f_{B}$ and $f_{B}^{*}$ which amount to about $30 \%$ and $20 \%$, respectively. Consequently, in the coupling $g_{B^{*} B \pi}$ itself, the NLO effects cancel almost completely as demonstrated in Fig. 1b. A similar cancellation takes place in the sum rule calculation of the $B \rightarrow \pi$ form factor $f^{+}$[2, 3]. As shown in [3], the origin of this cancellation can be understood by considering the heavy quark limit of the sum rules. In this limit, one has a partial cancellation of large $\ln \left(m_{b} / \omega_{0}\right)$ appearing in $f_{B} f^{+}$and $f_{B}$, where $\omega_{0}=\left(s_{0}^{B}-m_{b}^{2}\right) / 2 m_{b}$ determines the size of the duality interval which is mass-independent. In the present case, we observe a complete cancellation of these logarithms in the heavy-quark limit of the LCSR (22) and the 2SR for $f_{B} f_{B^{*}}$. As 
Table 1: Sum rule predictions for $B$ and $B^{*}$ mesons

\begin{tabular}{|c||c|c|}
\hline LCSR & $f_{B} f_{B^{*}} g_{B^{*} B \pi}$ & $0.80 \pm 0.23 \mathrm{GeV}^{2}$ \\
\hline 2SR & $f_{B}$ & $180 \pm 30 \mathrm{MeV}$ \\
\hline$\frac{\mathrm{LCSR}}{2 \mathrm{SR}}$ & $f_{B^{*}} g_{B^{*} B \pi}$ & $4.44 \pm 0.97 \mathrm{GeV}$ \\
\hline 2SR & $f_{B^{*}}$ & $195 \pm 35 \mathrm{MeV}$ \\
\hline$\frac{\mathrm{LCSR}}{2 \mathrm{SR}}$ & $g_{B^{*} B \pi}$ & $22 \pm 7$ \\
\hline
\end{tabular}

a result, the NLO correction to the coupling constant $g_{B^{*} B \pi}$ contains no logarithms of $m_{b}$ at all. Furthermore, the scale-dependence of $f_{B} f_{B^{*}} g_{B^{*} B \pi}$ is moderate. In LO, it only comes from the evolution of the distribution amplitude $\varphi_{\pi}$, while in NLO it also arises from the running of $\alpha_{s}(\mu)$. Making the usual choice $\mu=\sqrt{m_{B}^{2}-m_{b}^{2}}=2.4 \mathrm{GeV}$, a scale which is of the order of the Borel mass $M$, and allowing $\mu$ to vary from one half to twice of the above value, $f_{B} f_{B^{*}} g_{B^{*} B \pi}(\mathrm{NLO})$ varies by $10 \%$ relative to the nominal value. Again, this is comparable to the NLO scale-dependence of $f_{B}$ and $f_{B}^{*}$ so that $g_{B^{*} B \pi}$ is almost scale-independent as can be seen in Fig. $1 \mathrm{~b}$.

Similar features are observed in the $D$-meson case. In particular, the NLO correction to $f_{D} f_{D^{*}} g_{D^{*} D \pi}$ of roughly $15 \%$ is compensated by the NLO corrections to $f_{D}$ and $f_{D^{*}}$ of about $10 \%$ each. It may look surprising that the perturbative corrections are smaller in the charm case than in the beauty case, in contrast to the naive expectation from asymptotic freedom. The reason becomes again clear in the heavy quark limit: when going from beauty to charm, the growth of $\alpha_{s}(\mu)$ is overcompensated by the shrinkage of the above-mentioned logarithm $\ln \left(m_{b} / \omega_{0}\right)$.

The numerical predictions including the twist 3 and 4 contributions in LO [1] are summarized in Tables 1 and 2. In dividing out the pseudoscalar and/or vector meson decay constants from the LCSR results, one can follow different procedures. The simplest possibility is to divide the complete sum rule (22) by the NLO values of these constants. Alternatively, one may divide the twist 2 term in (22) by the NLO values of decay constants, while dividing the twist 3 and 4 terms by the LO values. The numerical difference lies within the overall theoretical uncertainty quoted in the Tables. We prefer the first option. For $g_{B^{*} B \pi}$, the uncertainty can be estimated as follows:

(a) Variation of the Borel parameters in the allowed ranges leads to a variation of the 
Table 2: Sum rule predictions for $D$ and $D^{*}$ mesons

\begin{tabular}{|c||c|c|}
\hline LCSR & $f_{D} f_{D^{*}} g_{D^{*} D \pi}$ & $0.54 \pm 0.15 \mathrm{GeV}^{2}$ \\
\hline $2 \mathrm{SR}$ & $f_{D}$ & $190 \pm 20 \mathrm{MeV}$ \\
\hline$\frac{\mathrm{LCSR}}{2 \mathrm{SR}}$ & $f_{D^{*}} g_{D^{*} D \pi}$ & $2.84 \pm 0.55 \mathrm{GeV}$ \\
\hline 2SR & $f_{D^{*}}$ & $270 \pm 35 \mathrm{MeV}$ \\
\hline$\frac{\mathrm{LCSR}}{2 \mathrm{SR}}$ & $g_{D^{*} D \pi}$ & $10.5 \pm 3$ \\
\hline
\end{tabular}

coupling by about $10 \%$.

(b) If the $b$-quark mass and the continuum threshold $s_{0}^{B}$ are varied simultaneously such that maximum stability of the sum rules for $f_{B}$ and $f_{B}^{*}$ is achieved, $g_{B^{*} B \pi}$ varies by $25 \%$.

(c) The uncertainty coming from the non-asymptotic terms in the pion distribution amplitudes amounts at most $10 \%$. This can be checked by discarding the non-asymptotic effects altogether.

(d) For the unknown NLO corrections beyond twist 2 we assign a $15 \%$ uncertainty. Since twist 3 contributes about $50 \%$ to the sum rule (22) this actually assumes an unknown correction to twist 3 and beyond as large as $30 \%$.

(e) The twist 4 contribution to the LCSR (22) is roughly $3 \%$. This can be taken as an indication of the uncertainty due to the neglect of twists higher than four.

(f) The size of the non-asymptotic terms in $\rho_{0}$ implies that they should affect the $O\left(\alpha_{s}\right)$ spectral density $\rho_{1}$ by less than $5 \%$.

(g) In (10) there is some ambiguity in the choice of the duality boundary $\widetilde{\Sigma}$ for $\rho_{1}$. A reasonable way to estimate the maximum uncertainty is to put the upper integration limit $s_{0}^{B}$ in (22) to infinity which corresponds to no continuum subtraction at all. The change is less than $1 \%$.

(h) The sensitivity to the precise value of the quark condensate density is rather small, because of a compensation of the effects in 2SR and in the twist 3 term of LCSR. The inaccuracy of higher-dimensional condensates in 2SR is unimportant.

Adding (a) to (h) in quadrature, the overall uncertainty on $g_{B^{*} B \pi}$ is shown in Table 1 together with the uncertainties in the related quantities estimated in a similar way. The corresponding error estimates for the $D$-meson case are listed in Table 2. 
A comprehensive comparison with predictions obtained by different methods can be found in Table 1 of ref. [1]. New estimates of $g_{B^{*} B \pi}$ in the framework of lattice QCD [15] and in a relativistic quark model [16] have appeared very recently. While our result shown in Table 1 agrees with the lattice estimate within uncertainties, it is smaller by a factor of 2-3 than the quark-model prediction.

5. As pointed out in the introduction, the $B^{*} B \pi$ matrix element determines the $B \rightarrow \pi$ form factor $f^{+}\left(p^{2}\right)$ near the kinematic limit $p^{2}=\left(m_{B}-m_{\pi}\right)^{2}$, where the $B^{*}$-pole dominates. More definitely, in the single-pole approximation, one has

$$
f^{+}\left(p^{2}\right)=\frac{f_{B^{*}} g_{B^{*} B \pi}}{2 m_{B^{*}}\left(1-p^{2} / m_{B^{*}}^{2}\right)}
$$

with the normalization $f_{B^{*}} g_{B^{*} B \pi}$ given in Table 1 . Since the NLO corrections to the approximation (26) at large $p^{2}$ and to $f^{+}\left(p^{2}\right)$ calculated from LCSR directly at low and intermediate $p^{2}$ are both small, the two descriptions match in NLO as well as they do in LO [回]. This also holds for the $D$-meson form factor.

Furthermore, from $g_{D^{*} D \pi}$ given in Table 2, one obtains :

$$
\Gamma\left(D^{*+} \rightarrow D^{0} \pi^{+}\right)=\frac{g_{D^{*} D \pi}^{2}}{24 \pi m_{D^{*}}^{2}}|\vec{q}|^{3}=23 \pm 13 \mathrm{keV}
$$

$\vec{q}$ being the decay 3-momentum. The current experimental limit 13

$$
\Gamma\left(D^{*+} \rightarrow D^{0} \pi^{+}\right)<89 \mathrm{keV}
$$

is still too high to challenge the theoretical prediction.

In conclusion, we have made some progress in the NLO analysis of the LCSR for the $B^{*} B \pi$ and $D^{*} D \pi$ coupling constants. The perturbative $O\left(\alpha_{s}\right)$-correction to the twist 2 term of this sum rule has been calculated removing one of the main uncertainties. The remaining uncertainties in $g_{B^{*} B \pi}$ and $g_{D^{*} D \pi}$ are mainly due to the heavy quark masses, the uncertainty in the pion distribution amplitudes of twist 2 and 3 at $u \simeq 1 / 2$, and the lack of the NLO corrections to the twist 3 contributions. Thus, there is still plenty of room for improvement.

\section{Acknowledgments}

We are grateful to V.M. Braun for useful discussions. This work was supported by the German Federal Ministry for Education and Research (BMBF) under contract number 05 7WZ91P (0). 


\section{References}

[1] V.M. Belyaev, V.M. Braun, A. Khodjamirian and R. Rückl, Phys. Rev. D51 (1995) 6177.

[2] A. Khodjamirian, R. Rückl, S. Weinzierl and O. Yakovlev, Phys. Lett. B410 (1997) 275 .

[3] E. Bagan, P. Ball and V.M. Braun, Phys. Lett. B417 (1998) 154.

[4] P. Ball and V.M. Braun, Phys. Rev. D58 (1998) 094016.

[5] P. Ball, JHEP (1998) 9809:005.

[6] A. Khodjamirian and R. Rückl, hep-ph/9801443, in Heavy Flavours II, eds. A.J. Buras and M. Lindner (World Scientific, Singapore,1998), p. 345.

[7] V.M. Braun, hep-ph/9801222, in Proc. of 4th International Workshop on Progress in Heavy Quark Physics, eds. M. Beyer, T. Mannel and H. Schröder (University of Rostock, 1998), p. 105.

[8] G.P. Lepage and S.J. Brodsky, Phys. Lett. B87 (1979) 359; Phys. Rev. D22 (1980) 2157 ;

S.J. Brodsky and G.P. Lepage, in: Perturbative Quantum Chromodynamics, ed. A.H. Mueller (World Scientific, Singapore, 1989) p. 93.

[9] V.L. Chernyak and A.R. Zhitnitsky, JETP Lett. 25 (1977) 510; Yad. Fiz. 31 (1980) 1053; Phys. Rep. 112 (1984) 173;

A.V. Efremov and A.V. Radyushkin, Phys. Lett. B94 (1980) 245; Teor. Mat. Fiz. 42 (1980) 147.

[10] F.M. Dittes and A.V. Radyushkin, Phys. Lett. B134 (1984) 359;

M.H. Sarmadi, Phys. Lett. B143 (1984) 471;

S.V. Mikhailov and A.V. Radyushkin, Nucl. Phys. B254 (1985) 89.

[11] E.P. Kadantseva, S.V. Mikhailov and A.V. Radyushkin, Sov. J. Nucl. Phys. 44 (1986) 326.

[12] T.M. Aliev and V.L. Eletsky, Sov. J. Nucl. Phys. 38 (1983) 936;

L.J. Reinders, H.Rubinstein and S. Yasaki, Phys. Rep. 127 (1985) 1.

[13] Particle Data Group (C. Caso et al.), Eur. Phys. J. C3 (1998) 1.

[14] V.M. Braun and I.B. Filyanov, Z. Phys. C44 (1989) 157.

[15] UKQCD Collaboration (G.M. de Divitiis et al.), JHEP (1998) 9810:010.

[16] D. Becirevic and A. Le Yaouanc, hep-ph/9901431. 